\section{Impacto do distanciamento social nas notificações de violência contra crianças e adolescentes no Rio Grande do Sul, Brasil}

\author{
Impact of social distancing on reports of violence \\ against children and adolescents in Rio Grande \\ do Sul, Brazil
}

\section{Impacto del confinamiento en las notificaciones de violencia contra niños y adolescentes en Río Grande do Sul, Brasil}

\author{
Mateus Luz Levandowski 1,2 \\ Douglas Nunes Stahnke 3 \\ Tiago N. Munhoz 1,2,4 \\ Jean Von Hohendorff 5 \\ Roberta Salvador-Silva 6
}

doi: 10.1590/0102-311X00140020

\title{
Resumo
}

O objetivo do estudo foi analisar as taxas de notificações de violência infantojuvenil no Estado do Rio Grande do Sul, Brasil, de 2015 a 2020 e as alterações em suas tendências por períodos devido à pandemia do novo coronavírus (COVID-19). É um estudo ecológico de séries temporais com dados secundários obtidos pelo Portal Bi Saúde no painel de Violência Interpessoal/Suicídio. Foram coletadas as notificações de violência em indivíduos de 0 a 19 anos, nos meses de março e abril de cada ano, estratificadas por sexo, faixa etária, raça/cor e tipo de violência. Utilizou-se a regressão de Prais-Winsten para a análise de tendência temporal. Das 7.718 notificações analisadas, observou-se uma queda de 54\% no ano de 2020 comparado com o mesmo período em 2019. A análise de tendência até 2019 indicou aumento nas taxas de notificações (2,04, IC95\%: 1,01; 3,07, $p=0,002)$, porém, com a inclusão do ano de 2020 à série temporal, o direcionamento na tendência das taxas de notificações inverteu-se para negativo, perdendo a significância estatística (-0,39, IC95\%: -1,16; $2,14, p=0,632$ ). Conclui-se que o distanciamento social devido à pandemia reduziu as taxas de notificações de violência contra crianças e adolescentes devido à subnotificação, exigindo estratégias que melhorem a identificação dos casos suspeitos de violência durante a pandemia. Ressalta-se a necessidade de planejamento e ações intersetoriais (como saúde, proteção social, justiça e segurança pública) rápidas e específicas com o objetivo da garantia dos direitos das crianças e dos adolescentes.

COVID-19; Isolamento Social; Maus-Tratos Infantis; Violência Doméstica; Pandemias

\author{
Correspondência \\ M. L. Levandowski \\ Curso de Psicologia, Universidade Federal de Pelotas. \\ Av. Ferreira Viana 3010, Pelotas, RS 96085-000, Brasil. \\ luzlevandowski@gmail.com \\ ${ }^{1}$ Curso de Psicologia, Universidade Federal de Pelotas, \\ Pelotas, Brasil. \\ 2 Programa de Pós-graduação em Psicologia, \\ Universidade Federal do Rio Grande, Rio Grande, Brasil. \\ 3 Universidade do Vale do Rio dos Sinos, São Leopoldo, Brasil. \\ 4 Programa de Pós-graduação em Epidemiologia, \\ Universidade Federal de Pelotas, Pelotas, Brasil. \\ 5 Programa de Pós-graduação em Psicologia, \\ Faculdade Meridional (IMED), Passo Fundo, Brasil. \\ ${ }^{6}$ Human Development and Violence Research Centre, \\ Universidade Federal de Pelotas, Pelotas, Brasil.
}




\section{Introdução}

A violência contra crianças e adolescentes inclui todas as formas de violência contra este grupo, sendo um problema social e de saúde pública em todo o mundo 1. Uma revisão com estudos de 96 países estimou que até 1 bilhão de crianças de 2 a 17 anos tenham sofrido algum tipo de violência (negligência, violência física, sexual ou psicológica) em 2014, destas, 100 milhões na América Latina 2. Embora a violência contra crianças e adolescentes seja um problema de saúde pública global, fatores geográficos e econômicos são capazes de influenciar as variações nas estimativas de violência 3 . Outros fatores também aumentam a susceptibilidade para a violência infantil. Austeridade econômica, desastres e emergências podem ser considerados fatores de risco para o aumento da violência contra grupos mais vulneráveis 4 .

Nesse contexto, no dia 30 de janeiro de 2020, foi declarada emergência global de Saúde Pública pela Organização Mundial da Saúde (OMS) em função do número de países atingidos pelo novo coronavírus (SARS-CoV-2), causador da doença COVID-19. Por causa do status de pandemia, adquirido em 11 de março ${ }^{5}$, muitos países começaram a adotar medidas de proteção contra a propagação do vírus. No Brasil, até o dia 23 de março, a maioria das unidades federativas já havia, pelo menos, limitado a abertura de serviços não essenciais, suspendendo as aulas e iniciando algum modelo de distanciamento social 6.

Embora o distanciamento social seja fundamental para diminuir a propagação exponencial do vírus 7 , muitas crianças e adolescentes podem estar enfrentando uma situação de risco aumentado. Isso acontece porque esses jovens estão mais tempo em casa com os seus agressores, já que cerca de 300 milhões de crianças no mundo são regularmente submetidas à violência física ou psicológica dentro da própria casa, segundo dados do relatório de 2017 da Fundo das Nações Unidas para a Infância (UNICEF) 8. No Brasil, os dados de 2018, do Ministério da Saúde, apontaram que 80\% dos casos de violência contra crianças e adolescentes ocorreram dentro de casa perpetrados por pais ou cuidadores 9 . Dessa forma, jovens que vivem com agressores estariam ainda mais expostos à violência durante as medidas de distanciamento social devido à pandemia do novo coronavírus. Além disso, outro aspecto preocupante das medidas protetivas contra o vírus é o fechamento das escolas. Essa medida é imprescindível para diminuir o ritmo de contágio do vírus na população, porém é no convívio com professores e outros profissionais do ambiente escolar que casos de violência contra crianças e adolescentes podem ser identificados e muitas vezes notificados aos órgãos competentes 10 . Para ilustrar, durante a epidemia do vírus ebola (de 2014 a 2016), nos países da África Ocidental, houve um aumento significativo dos casos de violência contra crianças e adolescentes comparado com os períodos anteriores à epidemia. Desses, a maior parte ocorreu em casa durante o período de fechamento das escolas ${ }^{8}$. Considerando essas informações, a Organização das Nações Unidas (ONU) faz um alerta para o aumento de violência doméstica que pode ocorrer em meio à quarentena imposta em resposta à pandemia 11.

Dessa maneira, a necessidade de distanciamento social e a consequente restrição de acesso às aulas e à circulação social configuram fatores de risco para a ocorrência e subnotificação de casos de violência contra crianças e adolescentes. A permanência em casa e a impossibilidade do convívio com um/a adulto/a de referência dificultam ainda mais a identificação dos casos de violência, bem como a busca por ajuda 12. Por exemplo, um aumento da violência contra mulher durante o período de isolamento foi detectado, o mesmo não ocorreu no caso da violência infantil 13. Assim, é necessário o acompanhamento das notificações de violência infantil, pois este é um instrumento de garantia de direitos importante na proteção às vítimas, especialmente em períodos excepcionais como o vivenciado atualmente. Portanto, o objetivo deste estudo foi investigar as taxas de notificações de violência à criança e ao adolescente durante o período de distanciamento social no Estado do Rio Grande do Sul, Brasil, e analisar a tendência nos coeficientes de notificações de violência contra este grupo de 2015 a 2020 nos meses de março e abril. Embora em períodos de crise as evidências apontem para um aumento do número de casos de violência a grupos vulneráveis, nossa hipótese foi a de que encontraríamos uma redução no número de notificações devido ao distanciamento social, caracterizando um cenário de subnotificações. 


\section{Método}

\section{Delineamento}

Trata-se de um estudo ecológico, em que foram apresentadas e analisadas as tendências das taxas de notificações de violência contra crianças e adolescentes $(n=6.790)$ e verificadas as suas associações por sexo, faixa etária, raça/cor e tipo de violência na população de 0 a 19 anos do Estado do Rio Grande do Sul, no período de 2015 a 2020, nos meses de março e abril de cada ano. A Figura 1 ilustra o delineamento do estudo.

\section{Figura 1}

Ilustração do delineamento do estudo.

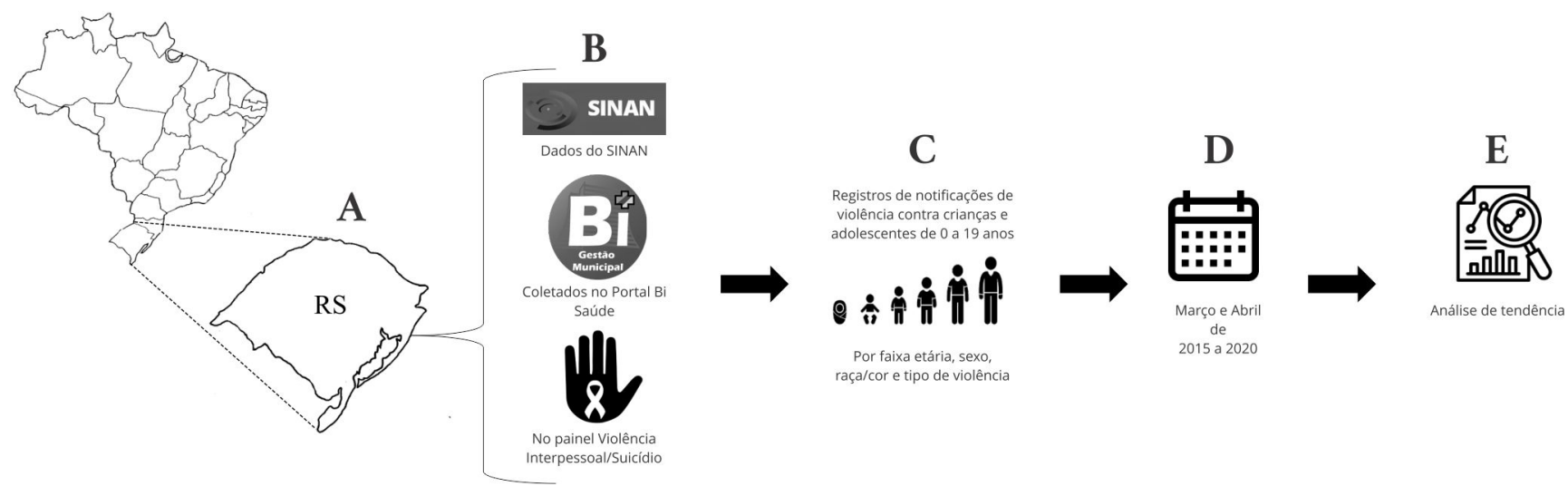

(A) Selecionado o Estado do Rio Grande do Sul; (B) dados do Sistema de Informação de Agravos de Notificação (SINAN) foram acessados no Portal Bi Saúde (http://bipublico.saude.rs.gov.br/index.htm) no painel Violência Interpessoal/Suicídio; (C) dois pesquisadores de maneira independente coletaram os dados de violência infantil por faixa etária, sexo, raça/cor e tipo de violência; (D) nos períodos de março e abril de 2015-2020; (E) para posteriores análises estatísticas.

\section{Coleta de dados}

As notificações de violência contra crianças e adolescentes foram coletadas utilizando-se dados secundários, disponibilizados pelo Departamento de Gestão de Tecnologia da Informação da Secretaria Estadual de Saúde do Estado do Rio Grande do Sul, Brasil, no Portal Business Intelligence Gestor Municipal (Portal Bi Saúde; http://bipublico.saude.rs.gov.br/index.htm). Para este estudo, foram usados os indicadores de Violência interpessoal/Suicídio compostos pelas notificações de violência interpessoal/autoprovocada do Sistema de Informação de Agravos de Notificação (SINAN).

Foi analisado o total de notificações por violência à criança e ao adolescente ( $\leq 19$ anos), estratificado por sexo (masculino e feminino), faixa etária (0-4, 5-9, 10-14 e 15-19 anos), raça/cor (branca, preta, parda, indígena e outras/não preenchido) e por tipo de violência (sexual, física, psicológica/moral, negligência/abandono, tortura, trabalho infantil/financeira e outras), nos meses de março e abril de cada ano do estudo. O número de notificações por tipo de violência pode ser superior ao número total de notificações, já que cada vítima pode ter sofrido mais de um tipo de violência por notificação. 
A faixa etária foi escolhida conforme a convenção elaborada pela OMS e adotada pelo Ministério da Saúde do Brasil 14. Essa convenção entende crianças como indivíduos de 0 a 9 anos e adolescentes aqueles de 10-19 anos. Os meses de março e abril foram selecionados, pois compreendem o período de distanciamento social no Estado do Rio Grande do Sul. A partir do dia 19 de março, houve a suspensão das atividades escolares na rede pública e privada de ensino (Decreto no 55.129 15) e foi declarado estado de calamidade pública em todo o território gaúcho (Decreto no 55.12816), iniciando a maior fase de distanciamento social. Já no dia 20 de março, foi restrita a abertura de serviços não essenciais para diminuir aglomerações (Decreto no 55.115 17). As medidas de prevenção contra o novo coronavírus permaneceram durante todo o mês de abril (Decretos no 55.154 18, no 55.177 19, no 55.184 20, no 55.22021). Já a série histórica iniciou em 2015 para cumprir requisitos mínimos para as análises estatísticas de tendência usadas neste estudo 22 .

A extração dos dados ocorreu entre os dias 6 de maio de 2020 e 14 de maio de 2020, de maneira duplicada e independente por dois pesquisadores para posterior conferência das informações. Para a criação do banco de dados foi utilizado o programa Microsoft Office Excel 2010 (https://products. office.com/).

\section{Tratamento dos dados}

Para a realização das taxas de notificação de violência contra crianças e adolescentes usou-se a razão entre o número de notificações de violência por estratificação no Rio Grande do Sul no ano/população exposta ao risco por estratificação no Rio Grande do Sul no ano, multiplicada por 100 mil. Já para o cálculo da população utilizada no denominador da equação da taxa de notificação de violência contra crianças e adolescentes por raça, foi calculada a porcentagem de cada raça na população do Rio Grande do Sul em cada ano do estudo, baseando-se na porcentagem destas raças conforme o Censo Demográfico de 2010. Os dados populacionais usados para a realização das taxas estavam disponíveis no site do Departamento de Informática do SUS (DATASUS; https://www2.datasus.gov.br), exceto para o extrato raça, obtidos pelo Sistema de Recuperação Automática (SIDRA), no site do Instituto Brasileiro de Geografia e Estatística (IBGE; https://www.ibge.gov.br/). Para o cálculo das taxas, foi utilizado o programa Microsoft Office Excel 2010.

\section{Análise estatística}

A tendência temporal foi analisada baseando-se nos modelos de regressão linear generalizada de Prais-Winsten, método no qual considera-se a auto correlação serial, fato comum em séries temporais, que pode superestimar as medidas de ajuste 22. Os resultados foram expressos por coeficientes que indicam aumento, estagnação ou diminuição da tendência com os seus correspondentes intervalos de 95\% de confiança (IC95\%). Baseado nesses coeficientes, realizou-se uma comparação entre os dois períodos do estudo (2015-2019 e 2015-2020), cujo resultado foi demonstrado como estável quando a tendência dos períodos manteve a direção ou inversão quando a tendência mudou a direção de um período para o outro. Para a análise descritiva foi usado o programa Graph Pad Prism versão 8.4 (https://www.graphpad.com/scientific-software/prism/) e para a análise de tendência foi utilizado o programa Stata versão 11.0 (https://www.stata.com). Adotou-se um nível de 5\% de significância $(\mathrm{p}<0,05)$. Todos os dados coletados são apresentados em formato de frequência bruta e taxa populacional em tabela e gráficos.

\section{Cuidados éticos}

Este estudo não foi registrado nem avaliado pelo sistema Comitê de Ética em Pesquisa/Comissão Nacional de Ética em Pesquisa, pois foram utilizados dados agregados de acesso público, não expondo nenhum ser humano à coleta ou intervenção. Os dados disponíveis no Portal Bi Saúde são de domínio público, obedecendo à Lei de Acesso à Informação e não apresentam dados individuais, ou seja, sem qualquer menção à identidade pessoal. 


\section{Resultados}

Os dados de 7.718 notificações foram descritos e analisados neste estudo. Os dados descritivos (frequência absoluta e taxa populacional) referentes as 7.718 notificações de violência contra crianças e adolescentes de todas as variáveis estudadas são apresentados na Tabela 1. Considerando-se as frequências absolutas das notificações de violência contra esse grupo de 2015-2020, houve predominância no sexo feminino, na faixa etária de 15-19 anos, na raça branca, sendo a violência física a mais frequente. Entretanto, nas notificações de violência por taxa populacional, os maiores valores estavam relacionados a meninas, de 15-19 anos e das raças indígena e preta. A violência física apresentou as maiores taxas de notificação seguida da negligência/abandono. Ainda, os valores mais altos dentro da série histórica para cada variável foram em abril de 2019, com exceção de violência física, psicológica/ moral, trabalho infantil e tortura, indicando que abril de 2019 foi o ápice de notificações dentro do período estudado.

A proporção da mudança anual do total de notificações entre os anos de 2015 e 2020 no bimestre de março/abril foi de: 0\% entre 2015-2016; aumento de 7\% entre 2016-2017; aumento de 52\% entre 2017-2018; aumento de 24\% entre 2018-2019; e redução de 54\% entre 2019-2020. Quando observada a proporção da mudança anual do total de notificações separadamente por mês, apenas entre 2019 e 2020, constata-se que: (i) com 38\% do tempo em regime de distanciamento social (março/2020) houve uma redução de $41 \%$ na taxa de notificações de violência comparando com março/2019; e (ii) com $100 \%$ do tempo em regime de distanciamento social (abril/2020) houve uma redução de $65 \%$ na taxa de notificações de violência comparando com abril/2019 (Figura 2). Na Figura 2 é ilustrada a taxa de notificações de violência, com destaque para os meses relacionados ao distanciamento social.

$\mathrm{Na}$ análise de tendência das taxas de notificações de violência contra crianças e adolescentes foram considerados os períodos de 2015-2019 e de 2015-2020 comparando-os entre si, com o intuito de observar a influência do distanciamento social na direção dos coeficientes (Tabela 2).

No período de 2015-2019, com exceção da tortura e da violência por negligência/abandono, todas as variáveis apresentaram coeficientes positivos, indicando uma tendência de aumento nas taxas de notificações no decorrer dos anos. Apenas três variáveis não demonstraram significância estatística: a faixa etária de 0-4 anos, a violência por negligência/abandono e o trabalho infantil.

Com a inclusão do ano de 2020 à série temporal, a direção da tendência dos coeficientes de notificações por violência contra crianças e adolescentes inverteu na comparação com o período até 2019, de crescente para decrescente, constatando-se que o ano de 2020 foi suficiente para a mudança dos resultados relacionados ao total de notificações, ao sexo, à faixa etária, à raça (apenas a branca) e por tipo de violência (exceto a violência psicológica/moral e tortura). 
Tabela 1

Frequências absolutas e taxas de notificações de violência infantil no Rio Grande do Sul, Brasil, de 2015 a 2020.

\begin{tabular}{|c|c|c|c|c|c|c|c|c|c|c|c|c|}
\hline & $\begin{array}{c}\text { Março/ } \\
2015\end{array}$ & $\begin{array}{c}\text { Abril/ } \\
2015\end{array}$ & $\begin{array}{c}\text { Março/ } \\
2016\end{array}$ & $\begin{array}{c}\text { Abril/ } \\
2016\end{array}$ & $\begin{array}{c}\text { Março/ } \\
2017\end{array}$ & $\begin{array}{l}\text { Abril/ } \\
2017\end{array}$ & $\begin{array}{c}\text { Março/ } \\
2018\end{array}$ & $\begin{array}{l}\text { Abril/ } \\
2018\end{array}$ & $\begin{array}{c}\text { Março/ } \\
2019\end{array}$ & $\begin{array}{c}\text { Abril/ } \\
2019\end{array}$ & $\begin{array}{c}\text { Março/ } \\
2020\end{array}$ & $\begin{array}{c}\text { Abril/ } \\
2020\end{array}$ \\
\hline & \multicolumn{12}{|c|}{$[n(\operatorname{tax} a)][n(\operatorname{tax} a)][n(\operatorname{tax} a)][n(\operatorname{tax} a)][n(\operatorname{tax} a)][n(\operatorname{tax} a)][n(\operatorname{tax} a)][n(\operatorname{tax} a)][n(\operatorname{tax} a)][n(\operatorname{tax} a)][n(\operatorname{tax} a)][n$} \\
\hline Total & $\begin{array}{c}513 \\
(16,6)\end{array}$ & $\begin{array}{c}455 \\
(14,7)\end{array}$ & $\begin{array}{c}517 \\
(17,0)\end{array}$ & $\begin{array}{c}436 \\
(14,4)\end{array}$ & $\begin{array}{c}516 \\
(17,3)\end{array}$ & $\begin{array}{c}489 \\
(16,4)\end{array}$ & $\begin{array}{c}785 \\
(26,7)\end{array}$ & $\begin{array}{c}721 \\
(24,6)\end{array}$ & $\begin{array}{c}838 \\
(29,0)\end{array}$ & $\begin{array}{l}1.007 \\
(34,9)\end{array}$ & $\begin{array}{c}487 \\
(17,1)\end{array}$ & $\begin{array}{c}354 \\
(12,5)\end{array}$ \\
\hline \multicolumn{13}{|l|}{ Sexo } \\
\hline Masculino & $\begin{array}{c}192 \\
(12,2)\end{array}$ & $\begin{array}{c}183 \\
(11,6)\end{array}$ & $\begin{array}{c}214 \\
(13,8)\end{array}$ & $\begin{array}{c}186 \\
(12,0)\end{array}$ & $\begin{array}{c}164 \\
(10,7)\end{array}$ & $\begin{array}{c}165 \\
(10,8)\end{array}$ & $\begin{array}{c}257 \\
(17,1)\end{array}$ & $\begin{array}{c}251 \\
(16,7)\end{array}$ & $\begin{array}{c}262 \\
(17,7)\end{array}$ & $\begin{array}{c}316 \\
(21,3)\end{array}$ & $\begin{array}{c}145 \\
(9,9)\end{array}$ & $\begin{array}{l}100 \\
(6,9)\end{array}$ \\
\hline Feminino & $\begin{array}{c}321 \\
(21,3)\end{array}$ & $\begin{array}{c}272 \\
(18,1)\end{array}$ & $\begin{array}{c}303 \\
(20,5)\end{array}$ & $\begin{array}{c}250 \\
(16,9)\end{array}$ & $\begin{array}{c}352 \\
(24,2)\end{array}$ & $\begin{array}{c}324 \\
(22,2)\end{array}$ & $\begin{array}{c}528 \\
(36,9)\end{array}$ & $\begin{array}{c}470 \\
(32,8)\end{array}$ & $\begin{array}{c}576 \\
(40,9)\end{array}$ & $\begin{array}{c}691 \\
(49,1)\end{array}$ & $\begin{array}{c}342 \\
(24,6)\end{array}$ & $\begin{array}{c}254 \\
(18,3)\end{array}$ \\
\hline \multicolumn{13}{|l|}{ Faixa etária (anos) } \\
\hline $0-4$ & $\begin{array}{c}127 \\
(18,6)\end{array}$ & $\begin{array}{c}117 \\
(17,2)\end{array}$ & $\begin{array}{c}126 \\
(18,7)\end{array}$ & $\begin{array}{c}96 \\
(14,3)\end{array}$ & $\begin{array}{c}107 \\
(16,1)\end{array}$ & $\begin{array}{c}97 \\
(14,6)\end{array}$ & $\begin{array}{c}160 \\
(24,4)\end{array}$ & $\begin{array}{c}126 \\
(19,2)\end{array}$ & $\begin{array}{c}148 \\
(22,8)\end{array}$ & $\begin{array}{c}176 \\
(27,1)\end{array}$ & $\begin{array}{c}78 \\
(12,1)\end{array}$ & $\begin{array}{c}59 \\
(9,2)\end{array}$ \\
\hline $5-9$ & $\begin{array}{c}92 \\
(12,6)\end{array}$ & $\begin{array}{c}85 \\
(11,6)\end{array}$ & $\begin{array}{c}82 \\
(11,4)\end{array}$ & $\begin{array}{c}83 \\
(11,6)\end{array}$ & $\begin{array}{c}78 \\
(11,0)\end{array}$ & $\begin{array}{c}61 \\
(8,6)\end{array}$ & $\begin{array}{c}146 \\
(21,0)\end{array}$ & $\begin{array}{c}111 \\
(16,0)\end{array}$ & $\begin{array}{c}111 \\
(16,2)\end{array}$ & $\begin{array}{c}138 \\
(20,2)\end{array}$ & $\begin{array}{c}60 \\
(8,9)\end{array}$ & $\begin{array}{c}56 \\
(8,3)\end{array}$ \\
\hline $10-14$ & $\begin{array}{c}126 \\
(15,5)\end{array}$ & $\begin{array}{c}127 \\
(15,6)\end{array}$ & $\begin{array}{c}115 \\
(14,5)\end{array}$ & $\begin{array}{c}99 \\
(12,5)\end{array}$ & $\begin{array}{c}132 \\
(17,1)\end{array}$ & $\begin{array}{c}123 \\
(15,9)\end{array}$ & $\begin{array}{c}201 \\
(26,6)\end{array}$ & $\begin{array}{c}198 \\
(26,2)\end{array}$ & $\begin{array}{c}217 \\
(29,3)\end{array}$ & $\begin{array}{c}283 \\
(38,3)\end{array}$ & $\begin{array}{c}129 \\
(17,8)\end{array}$ & $\begin{array}{c}87 \\
(12,0)\end{array}$ \\
\hline $15-19$ & $\begin{array}{c}168 \\
(19,6)\end{array}$ & $\begin{array}{c}126 \\
(14,7)\end{array}$ & $\begin{array}{c}194 \\
(22,8)\end{array}$ & $\begin{array}{c}158 \\
(18,6)\end{array}$ & $\begin{array}{c}199 \\
(23,6)\end{array}$ & $\begin{array}{c}208 \\
(24,7)\end{array}$ & $\begin{array}{c}278 \\
(33,5)\end{array}$ & $\begin{array}{c}286 \\
(34,5)\end{array}$ & $\begin{array}{c}362 \\
(44,3)\end{array}$ & $\begin{array}{c}410 \\
(50,2)\end{array}$ & $\begin{array}{c}220 \\
(27,3)\end{array}$ & $\begin{array}{c}152 \\
(18,9)\end{array}$ \\
\hline \multicolumn{13}{|l|}{ Raça/Cor } \\
\hline Branca & $\begin{array}{c}403 \\
(56,2)\end{array}$ & $\begin{array}{c}337 \\
(47,0)\end{array}$ & $\begin{array}{c}373 \\
(52,8)\end{array}$ & $\begin{array}{c}317 \\
(44,9)\end{array}$ & $\begin{array}{c}397 \\
(57,2)\end{array}$ & $\begin{array}{c}401 \\
(57,7)\end{array}$ & $\begin{array}{c}587 \\
(85,9)\end{array}$ & $\begin{array}{c}522 \\
(76,4)\end{array}$ & $\begin{array}{c}624 \\
(92,8)\end{array}$ & $\begin{array}{c}759 \\
(112,9)\end{array}$ & $\begin{array}{c}350 \\
(52,8)\end{array}$ & $\begin{array}{c}251 \\
(37,9)\end{array}$ \\
\hline Preta & $\begin{array}{c}29 \\
(56,4)\end{array}$ & $\begin{array}{c}35 \\
(68,1)\end{array}$ & $\begin{array}{c}34 \\
(67,2)\end{array}$ & $\begin{array}{c}32 \\
(63,3)\end{array}$ & $\begin{array}{c}29 \\
(113,8)\end{array}$ & $\begin{array}{c}27 \\
(106,0)\end{array}$ & $\begin{array}{c}56 \\
(223,3)\end{array}$ & $\begin{array}{c}49 \\
(195,6)\end{array}$ & $\begin{array}{c}55 \\
(222,8)\end{array}$ & $\begin{array}{c}76 \\
(307,9)\end{array}$ & $\begin{array}{c}38 \\
(156,3)\end{array}$ & $\begin{array}{c}20 \\
(82,3)\end{array}$ \\
\hline Indígena & $\begin{array}{c}4 \\
(92,9)\end{array}$ & $\begin{array}{c}3 \\
(69,6)\end{array}$ & $\begin{array}{c}0 \\
(0,0)\end{array}$ & $\begin{array}{c}2 \\
(47,2)\end{array}$ & $\begin{array}{c}3 \\
(140,5)\end{array}$ & $\begin{array}{c}4 \\
(187,3)\end{array}$ & $\begin{array}{c}4 \\
(190,3)\end{array}$ & $\begin{array}{c}5 \\
(237,9)\end{array}$ & $\begin{array}{c}8 \\
(386,6)\end{array}$ & $\begin{array}{c}8 \\
(386,6)\end{array}$ & $\begin{array}{c}5 \\
(245,3)\end{array}$ & $\begin{array}{c}7 \\
(343,5)\end{array}$ \\
\hline Parda & $\begin{array}{c}58 \\
(49,2)\end{array}$ & $\begin{array}{c}50 \\
(42,4)\end{array}$ & $\begin{array}{c}70 \\
(60,3)\end{array}$ & $\begin{array}{c}56 \\
(48,3)\end{array}$ & $\begin{array}{c}60 \\
(102,7)\end{array}$ & $\begin{array}{c}37 \\
(63,3)\end{array}$ & $\begin{array}{c}77 \\
(133,9)\end{array}$ & $\begin{array}{c}97 \\
(168,7)\end{array}$ & $\begin{array}{c}96 \\
(169,6)\end{array}$ & $\begin{array}{c}100 \\
(176,6)\end{array}$ & $\begin{array}{c}63 \\
(113,0)\end{array}$ & $\begin{array}{c}49 \\
(87,9)\end{array}$ \\
\hline \multicolumn{13}{|l|}{ Tipo de violência } \\
\hline Sexual & $\begin{array}{l}119 \\
(3,8)\end{array}$ & $\begin{array}{l}116 \\
(3,7)\end{array}$ & $\begin{array}{l}120 \\
(3,9)\end{array}$ & $\begin{array}{l}106 \\
(3,5)\end{array}$ & $\begin{array}{l}128 \\
(4,3)\end{array}$ & $\begin{array}{l}128 \\
(4,3)\end{array}$ & $\begin{array}{l}230 \\
(7,8)\end{array}$ & $\begin{array}{l}186 \\
(6,3)\end{array}$ & $\begin{array}{l}183 \\
(6,3)\end{array}$ & $\begin{array}{l}204 \\
(7,0)\end{array}$ & $\begin{array}{l}117 \\
(4,1)\end{array}$ & $\begin{array}{c}85 \\
(3,0)\end{array}$ \\
\hline Física & $\begin{array}{l}238 \\
(7,7)\end{array}$ & $\begin{array}{l}185 \\
(6,0)\end{array}$ & $\begin{array}{l}244 \\
(8,0)\end{array}$ & $\begin{array}{l}223 \\
(7,3)\end{array}$ & $\begin{array}{l}213 \\
(7,1)\end{array}$ & $\begin{array}{l}212 \\
(7,1)\end{array}$ & $\begin{array}{l}256 \\
(8,7)\end{array}$ & $\begin{array}{l}263 \\
(8,9)\end{array}$ & $\begin{array}{l}227 \\
(7,8)\end{array}$ & $\begin{array}{l}228 \\
(7,9)\end{array}$ & $\begin{array}{l}133 \\
(4,6)\end{array}$ & $\begin{array}{l}100 \\
(3,5)\end{array}$ \\
\hline $\begin{array}{l}\text { Negligência/ } \\
\text { Abandono }\end{array}$ & $\begin{array}{l}163 \\
(5,3)\end{array}$ & $\begin{array}{l}146 \\
(4,7)\end{array}$ & $\begin{array}{l}179 \\
(5,9)\end{array}$ & $\begin{array}{l}124 \\
(4,1)\end{array}$ & $\begin{array}{l}137 \\
(4,6)\end{array}$ & $\begin{array}{l}121 \\
(4,0)\end{array}$ & $\begin{array}{l}164 \\
(5,5)\end{array}$ & $\begin{array}{c}140 \\
(4,78)\end{array}$ & $\begin{array}{l}179 \\
(6,2)\end{array}$ & $\begin{array}{l}211 \\
(7,3)\end{array}$ & $\begin{array}{c}82 \\
(2,8)\end{array}$ & $\begin{array}{c}65 \\
(2,3)\end{array}$ \\
\hline $\begin{array}{l}\text { Psicológica/ } \\
\text { Moral }\end{array}$ & $\begin{array}{l}166 \\
(5,4)\end{array}$ & $\begin{array}{l}127 \\
(4,1)\end{array}$ & $\begin{array}{c}131 \\
(4,3)\end{array}$ & $\begin{array}{l}114 \\
(3,7)\end{array}$ & $\begin{array}{c}125 \\
(4,2)\end{array}$ & $\begin{array}{c}101 \\
(3,3)\end{array}$ & $\begin{array}{c}106 \\
(3,6)\end{array}$ & $\begin{array}{c}67 \\
(2,2)\end{array}$ & $\begin{array}{c}35 \\
(1,2)\end{array}$ & $\begin{array}{c}47 \\
(1,6)\end{array}$ & $\begin{array}{c}29 \\
(1,0)\end{array}$ & $\begin{array}{c}19 \\
(0,7)\end{array}$ \\
\hline Trabalho infantil & $\begin{array}{c}7 \\
(0,2)\end{array}$ & $\begin{array}{c}8 \\
(0,2)\end{array}$ & $\begin{array}{c}3 \\
(0,1)\end{array}$ & $\begin{array}{c}0 \\
(0,0)\end{array}$ & $\begin{array}{c}5 \\
(0,1)\end{array}$ & $\begin{array}{c}6 \\
(0,2)\end{array}$ & $\begin{array}{c}12 \\
(0,4)\end{array}$ & $\begin{array}{c}5 \\
(0,1)\end{array}$ & $\begin{array}{c}3 \\
(0,1)\end{array}$ & $\begin{array}{c}10 \\
(0,3)\end{array}$ & $\begin{array}{c}6 \\
(0,2)\end{array}$ & $\begin{array}{l}100 \\
(3,5)\end{array}$ \\
\hline Tortura & $\begin{array}{c}12 \\
(0,3)\end{array}$ & $\begin{array}{c}10 \\
(0,3)\end{array}$ & $\begin{array}{c}14 \\
(0,4)\end{array}$ & $\begin{array}{c}4 \\
(0,1)\end{array}$ & $\begin{array}{c}8 \\
(0,2)\end{array}$ & $\begin{array}{c}8 \\
(0,2)\end{array}$ & $\begin{array}{c}4 \\
(0,1)\end{array}$ & $\begin{array}{c}10 \\
(0,3)\end{array}$ & $\begin{array}{c}3 \\
(0,1)\end{array}$ & $\begin{array}{c}2 \\
(0,0)\end{array}$ & $\begin{array}{c}1 \\
(0,0)\end{array}$ & $\begin{array}{c}2 \\
(0,07)\end{array}$ \\
\hline Outros & $\begin{array}{c}32 \\
(1,0)\end{array}$ & $\begin{array}{c}35 \\
(1,1)\end{array}$ & $\begin{array}{c}37 \\
(1,2)\end{array}$ & $\begin{array}{c}34 \\
(1,1)\end{array}$ & $\begin{array}{c}67 \\
(2,2)\end{array}$ & $\begin{array}{c}73 \\
(2,4)\end{array}$ & $\begin{array}{l}130 \\
(4,4)\end{array}$ & $\begin{array}{l}135 \\
(4,6)\end{array}$ & $\begin{array}{l}225 \\
(7,8)\end{array}$ & $\begin{array}{c}322 \\
(11,1)\end{array}$ & $\begin{array}{l}145 \\
(5,1)\end{array}$ & $\begin{array}{c}3 \\
(0,1)\end{array}$ \\
\hline
\end{tabular}

Nota: taxa populacional por 100 mil habitantes. 


\section{Figura 2}

Taxa de notificações de violência infantil em março e abril de 2015 a 2020 no Rio Grande do Sul, Brasil.

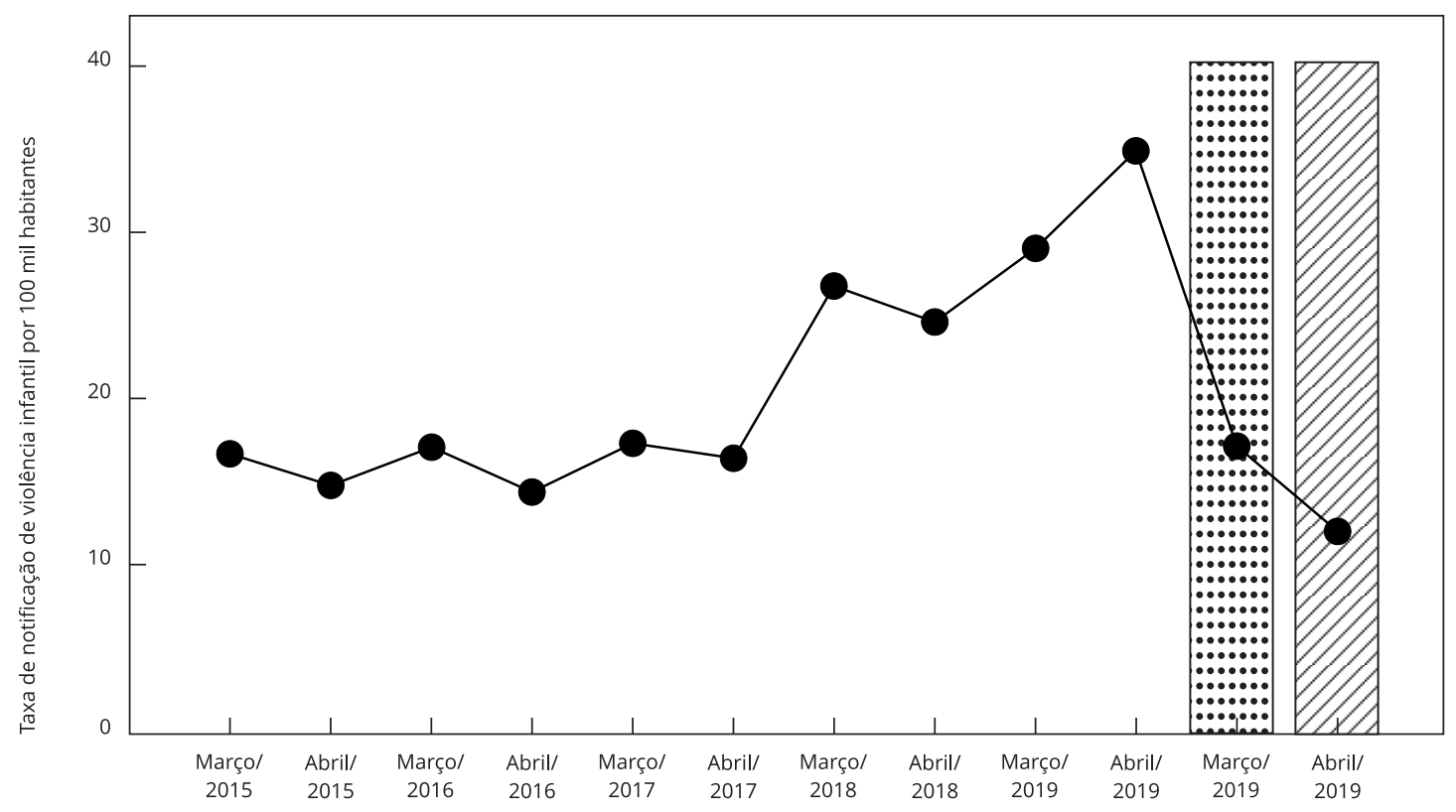

Nota: é demonstrada a taxa do total de notificações de violência infantil por 100 mil habitantes, com destaque para os meses relacionados ao distanciamento social devido ao novo coronavírus. Coluna com pontilhados indica que 38\% do mês em regime de distanciamento social e suspensão de aulas. Coluna com listras indica que $100 \%$ do mês em regime de distanciamento social e suspensão de aulas.

\section{Discussão}

Foi realizado um estudo ecológico de séries temporais para investigar os efeitos do distanciamento social nas notificações de violência contra crianças e adolescentes no Rio Grande do Sul. Utilizandose a análise de regressão linear generalizada de Prais-Winsten verificamos que houve um aumento significativo de notificações no período de 2015-2019, e a inclusão do ano de 2020 à série temporal (2015-2020) inverteu a direção da tendência temporal, ou seja, a inclusão do ano de 2020, o ano da pandemia do novo coronavírus, foi suficiente para demonstrar um decréscimo das notificações na série temporal. Além disso, comparando apenas o bimestre março/abril de 2020 (meses de início do distanciamento social no Rio Grande do Sul) com o mesmo período em 2019, observou-se uma redução de $54 \%$ na frequência de notificações.

Nossos resultados sobre o aumento das notificações entre 2015-2019 no estado estão de acordo com o último boletim epidemiológico do Ministério da Saúde entre os anos de 2011-2018, mostrando aumento de $83 \%$ nas notificações de violência sexual infantil em todo o território nacional, especialmente nas regiões Sudeste e Sul 9 . Esse aumento das notificações é visto em outros estudos 12 e está associado à implementação da notificação compulsória de casos de violência em 2011, conjuntamente com ações de formação de profissionais na prevenção e enfrentamento à violência infantil 23 . Em diferentes países das Américas e Europa há evidências do aumento de 30\% nas notificações de situações de violência doméstica contra meninas e mulheres ${ }^{24}$. O necessário distanciamento social para a mitigação do impacto do novo coronavírus afeta a renda e as condições de trabalho da população, com prejuízos ainda mais acentuados sobre as populações mais vulneráveis 24 .

O presente estudo identificou uma queda no número de notificações no período de março e abril de 2020. Esse achado deve ser cuidadosamente interpretado, uma vez que reflete a redução da iden- 
Tabela 2

Coeficientes de tendência das taxas estratificadas de notificações de violência infantil no Rio Grande do Sul, Brasil, por períodos.

\begin{tabular}{|c|c|c|c|c|c|c|c|}
\hline & \multicolumn{3}{|c|}{ 2015-2019 } & \multicolumn{3}{|c|}{$2015-2020$} & \multirow{2}{*}{$\begin{array}{c}\text { Comparação das } \\
\text { tendências ** }\end{array}$} \\
\hline & Coeficiente * & IC95\% & Valor de $p$ & Coeficiente * & IC95\% & Valor de $p$ & \\
\hline Total & 2,04 & 1,$01 ; 3,07$ & 0,002 & $-0,39$ & $-1.36 ; 2,14$ & 0,632 & Inversão \\
\hline \multicolumn{8}{|l|}{ Sexo } \\
\hline Masculino & 0,95 & 0,$22 ; 1,69$ & 0,017 & $-0,60$ & $-2,30 ; 1,08$ & 0,441 & Inversão \\
\hline Feminino & 3,20 & 1,$8 ; 4,60$ & 0,001 & $-0,61$ & $-4,68 ; 3,45$ & 0,743 & Inversão \\
\hline \multicolumn{8}{|l|}{ Faixa etária (anos) } \\
\hline $0-4$ & 0,82 & $-0,79 ; 2,36$ & 0,253 & $-0,95$ & $-2,84 ; 0,93$ & 0,285 & Inversão \\
\hline $5-9$ & 0,91 & 0,$19 ; 1,62$ & 0,018 & $-0,41$ & $-1,87 ; 1,04$ & 0,543 & Inversão \\
\hline $10-14$ & 2,45 & 1,$06 ; 3,85$ & 0,004 & $-0,40$ & $-3,46 ; 2,64$ & 0,772 & Inversão \\
\hline $15-19$ & 3,53 & 2,$23 ; 4,83$ & $<0,001$ & $-0,60$ & $-5,01 ; 3,80$ & 0,767 & Inversão \\
\hline \multicolumn{8}{|l|}{ Raça/cor } \\
\hline Branca & 6,48 & 3,$29 ; 9,66$ & 0,002 & $-2,24$ & $-11,45 ; 6,96$ & 0,599 & Inversão \\
\hline Preta & 26,98 & 18,$72 ; 35,23$ & $<0,001$ & 3,81 & $-21,44 ; 29,07$ & 0,743 & Estável \\
\hline Indígena & 37,35 & 18,$24 ; 57,22$ & 0,002 & 9,30 & $-23,17 ; 41,78$ & 0,538 & Estável \\
\hline Parda & 17,20 & 11,$97 ; 22,42$ & $<0,001$ & 0,65 & $-17,22 ; 18,53$ & 0,936 & Estável \\
\hline \multicolumn{8}{|l|}{ Tipo de violência } \\
\hline Sexual & 0,43 & 0,$20 ; 0,66$ & 0,002 & $-0,16$ & $-0,83 ; 0,50$ & 0,592 & Inversão \\
\hline Física & 0,15 & 0,$00 ; 0,31$ & 0,047 & $-0,56$ & $-1,32 ; 0,19$ & 0,130 & Inversão \\
\hline Negligência/Abandono & 0,16 & $-0,08 ; 0,41$ & 0,175 & $-0,27$ & $-0,74 ; 0,20$ & 0,229 & Inversão \\
\hline Psicológica/Moral & $-0,38$ & $-0,53 ;-0,26$ & $<0,001$ & $-0,43$ & $-0,53 ;-0,33$ & $<0,001$ & Estável \\
\hline Trabalho infantil & 0,00 & $-0,02 ; 0,04$ & 0,501 & $-0,00$ & $-0,02 ; 0,02$ & 0,854 & Inversão \\
\hline Tortura & $-0,02$ & $-0,04 ;-0,01$ & 0,002 & $-0,03$ & $-0,04 ;-0,02$ & $<0,001$ & Estável \\
\hline
\end{tabular}

IC95\%: intervalo de 95\% de confiança.

* Coeficiente de regressão linear generalizada de Prais-Winsten;

** Comparação qualitativa dos coeficientes de 2015-2019 com os coeficientes de 2015-2020. É apresentado como Estável quando o coeficiente se manteve positivo ou negativo nas duas análises e apresentado como Inversão quando o coeficiente mudou de positivo para negativo.

tificação e notificação dos casos de violência e não a redução da ocorrência de situações de violência contra crianças e adolescentes. Evidências apontam que períodos de crise e desastres aumentam o número de casos de violência à criança e ao adolescente, como nos casos do furacão Floyd 25, furacão Hugo 26, terremoto Loma Prieta 26 nos Estados Unidos e a epidemia de ebola na África Ocidental 8. Nesse sentido, um estudo experimental e longitudinal na Libéria, durante o surto de ebola, identificou aumento de preferência por práticas parentais punitivas severas, como agressões físicas e verbais 27. Essas evidências apontam que crises e desastres podem aumentar os casos de violência contra crianças e adolescentes em função do estresse adicional conferido pelo evento, como sensação de incerteza, mortes e prejuízos financeiros 28 .

Outro aspecto importante a ressaltar é que os dados demonstram marcada desigualdade racial. Nesse sentido, uma revisão sistemática sobre racismo e seus efeitos na saúde da população ressalta a importância do aprofundamento e investigação do tema 29. Soma-se a isso os dados da Pesquisa Nacional de Saúde que indicaram maior ocorrência de vitimização por violência por pessoa conhecida entre os indivíduos adultos autodeclarados negros, e quase duas vezes maior risco de lesão corporal do que as pessoas brancas 30 .

Evidências sobre o distanciamento social e a ocorrência de violência durante a pandemia do novo coronavírus ainda são escassas ${ }^{31}$. No entanto, sabe-se que a identificação e encaminhamento dos casos de violência ocorrem, muitas vezes, no ambiente escolar 10. Nos Estados Unidos, 20,5\% das notificações de violência contra crianças e adolescentes foram realizadas por educadores, notando-se uma flutuação sazonal com menos notificações no período de férias escolares 32. Já no Brasil, um estudo identificou que $28,6 \%$ das notificações de abuso sexual contra meninos foram realizadas pela 
escola 33. Nesse sentido, o Ministério da Educação fornece um guia para que educadores possam identificar sinais emitidos de violência pela criança e assim notificar e proteger 34. Além disso, o ambiente escolar favorece estratégias de coping para crianças e adolescentes 35 , bem como oportunidade para o estabelecimento de relações interpessoais com seus pares. Também favorece o contato com adultos com potencial habilidade de cuidado e manejo adequado de situações de violência, incluindo o suporte necessário e o encaminhamento aos serviços de saúde e proteção social 26,36.

Algumas informações apontam para um aumento do número real de casos de violência infantil e a subnotificação também pode aumentar em períodos de crise. Com o avanço da pandemia do novo coronavírus, a necessidade do distanciamento social e do fechamento das escolas e de outros serviços, a atenção e o cuidado voltados para crianças e adolescentes tendem a diminuir. A queda das notificações observada neste estudo, em vez de representar uma redução dos casos de violência contra crianças e adolescentes, provavelmente reflete uma queda da identificação e notificação dos casos existentes.

Um estudo sobre os impactos da pandemia do novo coronavírus sobre a ocorrência de crimes nos Estados Unidos reportou uma diminuição na ocorrência de crimes violentos e um aumento nas notificações de violência doméstica no mesmo período 37 . Estudos recentes acerca dos efeitos da pandemia sobre a saúde mental e a ocorrência de violência doméstica ou contra crianças e adolescentes ainda são incipientes, e a magnitude e/ou determinantes das situações de violência ainda não foram claramente identificados 38 .

Medidas emergenciais têm sido propostas com foco no enfrentamento das situações de violência doméstica durante a pandemia do novo coronavírus. Dentre elas, destacam-se aquelas aplicáveis aos casos contra crianças e adolescentes descritas por Marques et al. 28, tais como: (1) a garantia de atendimento 24 horas pelos canais de notificação, como o Disque 100; (2) manutenção do trabalho dos Conselhos Tutelares, seja por plantão presencial ou via telefone, WhatsApp e aplicativos de celulares; (3) incremento de campanhas de alerta acerca das situações de violência contra crianças e adolescentes, principalmente aquelas voltadas a vizinhos, parentes e amigos 28 , que podem ter algum contato com prováveis vítimas e notificar os casos; (4) aumento do investimento em organizações civis que prestam auxílio às vítimas; (5) criação de sistemas de alerta em farmácias e mercados; (6) declaração de casas de acolhimento às vítimas como serviço essencial ${ }^{39}$. Além dessas medidas, acreditamos que campanhas direcionadas a professores incentivando a realização de notificação sejam necessárias. Professores que tinham suspeitas de violências contra seus alunos no período anterior ao distanciamento social, mas que não realizaram a notificação, devem ser incentivados a realizá-la atualmente. Além dessas campanhas, cursos online sobre o enfrentamento de situações de violência em períodos de crise poderiam ser oferecidos visando a preparar os profissionais para sua atuação. Ademais, é importante que os profissionais que estejam em serviço tenham sua integridade física assegurada por meio da disponibilização dos equipamentos de proteção individual.

$\mathrm{O}$ enfrentamento das situações de violência durante a pandemia por meio de medidas emergenciais tem ocorrido em diferentes países. Canadá, França, Austrália e Reino Unido investiram na ampliação da oferta de serviços, financiamento e apoio institucional dos entes governamentais. Serviços de abrigo para meninas e mulheres que sofreram violência foram considerados serviços essenciais no Canadá. Na Itália, os perpetradores de violência doméstica ou violência à criança e ao adolescente foram legalmente obrigados a deixar o domicílio no qual conviviam com as vítimas nos últimos meses. Na França, além do aumento da capacidade dos abrigos, o governo destinou hospedagem em hotéis para vítimas de violência. $\mathrm{Na}$ China, uma campanha pela Internet (\#AntiDomesticViolenceDurin gEpidemic) facilitou o acesso a orientações e autoridades responsáveis para o manejo e a condução dos casos de violência. Na África do Sul, grupos populacionais mais vulneráveis receberam atenção especial das instituições e serviços de saúde, com ações educativas e contato regular para a identificação de situações de riscos de violência 24 .

O Brasil conta com uma rede de proteção à criança e ao adolescente composta por diversos serviços. O serviço responsável por garantir os direitos da criança e do adolescente é o Conselho Tutelar, que deve, preferencialmente, receber as notificações de casos de suspeita ou confirmação de violência a esse grupo, visando a expedir medidas protetivas e os encaminhamentos necessários 40 para o atendimento no Sistema Único de Saúde (SUS) e de Sistema Único de Assistência Social (SUAS). No âmbito do SUS, a criança deve receber os cuidados em saúde física e mental, e no âmbito do SUAS 
devem ser realizados os atendimentos psicossociais e, quando necessário, o acolhimento institucional por meio da proteção social especial 41 . Além desses serviços, a rede é composta por todas as outras instituições que prestam algum atendimento à criança e ao adolescente, tal como a escola. A escola tem um papel fundamental no enfrentamento da violência, seja por ser espaço privilegiado para ações de prevenção, seja pelo contato sistemático com crianças e adolescentes, permitindo a identificação de possíveis casos de violência.

Estudos vêm mostrando as fragilidades nessa rede de proteção devido a fatores como a complexidade dos casos atendidos, a falta de condições estruturais ideais, de capacitação profissional e de articulação entre os serviços 42,43,44,45. Sendo assim, é esperado que, num momento de crise como a pandemia do novo coronavírus, tais fragilidades sejam exacerbadas, dificultando ainda mais a garantia de direitos de crianças e adolescentes, como por exemplo a demora entre a notificação e as medidas de proteção efetivas, podendo levar a perpetuação ou intensificação da violência. Para além das medidas emergências requeridas pela atual pandemia, é necessário investimento constante no aperfeiçoamento dos serviços da rede de proteção. Dentre esses investimentos, o mais premente é a capacitação profissional. É necessário que os profissionais estejam preparados tecnicamente para a identificação de casos de violência e para a realização da notificação. A falta de conhecimento dos procedimentos necessários para que a notificação seja realizada é indicada como impedimento para sua realização pelos profissionais 46 . Infelizmente, o que se tem presenciado no país, nos últimos anos, é a falta de investimento em serviços públicos 47 , o que acaba por gerar uma precarização no SUAS e no SUS, por exemplo. Além disso, a remuneração de professores do ensino público é defasada e o investimento em capacitação profissional não é suficiente.

Recentemente, foi aprovada a Lei no 13.431/2017 48, que "Estabelece o sistema de garantia de direitos da criança e do adolescente vítima ou testemunha de violência e altera a Lei no 8.069, de 13 de julho de 1990 (Estatuto da Criança e do Adolescente)". Dentre outros aspectos, essa Lei visa a tornar o sistema de garantia de direitos da criança e do adolescente mais articulado e coeso com vistas a oferecer intervenções mais efetivas aos usuários. Para que isso seja alcançado, de acordo com o Decreto que regulamenta tal lei, após 180 dias de sua publicação, deveria ser instituído, "preferencialmente no âmbito dos conselhos de direitos das crianças e dos adolescentes, o comitê de gestão colegiada da rede de cuidado e de proteção social das crianças e dos adolescentes vítimas ou testemunhas de violência, com a finalidade de articular, mobilizar, planejar, acompanhar e avaliar as ações da rede intersetorial, além de colaborar para a definição dos fluxos de atendimento e o aprimoramento da integração do referido comitê". Embora ainda não encontre-se estudos a respeito da criação de tais comitês, o que percebe-se é a sua ausência nos municípios. Caso existissem, tais comitês, numa situação de crise como a vivenciada pela atual pandemia poderiam ser acionados, em nível municipal, para coordenar ações emergências de enfrentamento da violência à criança e ao adolescente.

A resposta para o enfrentamento das situações de violência doméstica e de gênero deve incluir ações e estratégias articuladas entre diferentes níveis e esferas de governo e sociedade civil organizada 49. Dessa maneira, incluir a oferta acessível (digital e online) e considerar como essencial a manutenção de serviços no âmbito jurídico, bem como a inclusão de organizações de mulheres na articulação de políticas e programas de enfrentamento, implementação e monitoramento das situações de violência 46 . A experiência da China (com campanhas online) poderá auxiliar a implementação de estratégias de disseminação de informações e facilitação de acesso à rede especializada de cuidado de forma online e integrada entre diferentes setores, como justiça, saúde e proteção social. No entanto, em países com grande desigualdade socioeconômica, onde há restrição no acesso e qualidade de serviços digitais e online, existe um desafio no planejamento e operacionalização de estratégias de enfrentamento à violência contra mulheres, crianças e adolescentes. Uma alternativa complementar aos serviços online, principalmente para acessar famílias em situação de maior vulnerabilidade social, é a Estratégia Saúde da Família (ESF), que é composta por uma equipe multiprofissional. As equipes estão vinculadas às unidades básicas de saúde e são responsáveis por realizar visitas domiciliares às famílias, sendo a principal porta de entrada para o SUS. As equipes de Saúde da Família poderiam exercer um papel crucial na identificação e posterior notificações e encaminhamentos dos casos de suspeita de violência contra crianças e adolescentes por terem acesso ao contexto familiar no ambiente doméstico. Esse Programa, por já estar estabelecido na comunidade, poderia ser fortalecido e ampliado em uma situação de pandemia. 
O investimento em pesquisa e inovação torna-se fundamental e respostas rápidas precisam ser definidas. Localmente, uma resposta rápida e que tem sido adotada em outros países 46 inclui a adaptação e expansão dos serviços de proteção como os Centros de Referência em Assistência Social (CRAS e CREAS), além dos abrigos para aquelas em situação de violência. A vulnerabilidade social é um desafio para ações e intervenções de programas e políticas públicas. Entretanto, ações e estratégias de monitoramento à distância (via telefone e/ou algum dispositivo de troca de mensagens ou rede social) das famílias, bem como a disponibilização e ampliação dos canais de notificação, suporte e atendimento à distância poderão ser uma estratégia efetiva, especialmente durante este período de distanciamento social. Essas ações e estratégias precisam ser avaliadas pelos gestores locais na busca de soluções criativas, rápidas e efetivas para o enfrentamento dos casos de violência, especialmente durante o período de distanciamento social. Buscar a articulação intersetorial (educação, saúde e sistema de justiça, por exemplo) poderia facilitar a identificação de mulheres, crianças e adolescentes em maior risco para situações de violência e assim a oferta de manejo adequado destas situações. Além disso, a oferta de suporte psicossocial e manejo adequado das situações de violência poderiam ser organizados e ofertados em curto prazo, desde que seja considerada uma prioridade e sejam investidos recursos humanos e financeiros. Como já destacado, o fortalecimento, a capacitação e a ampliação do quadro de trabalhadores especializados nos setores saúde, assistência social e segurança pública são fundamentais para o enfrentamento dos casos de violência, durante e após a pandemia. O estabelecimento de parcerias com universidades, por exemplo, também poderia auxiliar na busca e implantação de ações.

Entre as limitações do estudo estão o delineamento ecológico, que tem como unidade de análise os grupos, não indivíduos, bem como aspectos relacionados à utilização de dados secundários, que estão sujeitos a erros de registro ou subnotificação. No entanto, é plausível considerar que tais limitações não comprometam o achado principal do estudo. Devido à indisponibilidade de dados atualizados em relação à raça/cor para o estado, a taxa de notificação de violência contra crianças e adolescentes por raça foi estimada com base no Censo Demográfico de 2010. Assim, assume-se que a estrutura racial da população não tenha sido alterada de forma significativa na última década. Destaca-se que a extração dos dados ocorreu por dupla digitação, contribuindo para a diminuição de erros na construção do banco de dados, e os critérios rigorosos para a realização das taxas de notificações estratificadas contribuem para a qualidade dos achados. O método escolhido - análise da tendência temporal - é considerado adequado para a correção de erros provenientes de autocorrelação em séries temporais. Por fim, embora alguns artigos acerca de violência e COVID-19 já tenham sido publicados, até onde temos conhecimento este é o primeiro estudo empírico sobre violência e COVID-19 no Brasil.

\section{Considerações finais}

Apesar de informações sobre o aumento nos casos de violência doméstica em diferentes países do mundo, o presente estudo explicita a redução de $70 \%$ na notificação dos casos de violência contra crianças e adolescentes durante o período de maior índice de distanciamento social no Rio Grande do Sul (março e abril de 2020). Dessa forma, fornece evidências sobre os efeitos do distanciamento social na ocorrência das notificações de violência entre crianças e adolescentes, ressaltando a necessidade de planejamento e ações intersetoriais (como saúde, proteção social, justiça e segurança pública) rápidas e específicas com o objetivo da garantia dos direitos deste grupo de indivíduos. Novos estudos em demais estados e com outras populações (mulheres e idosos, por exemplo) podem fornecer mais evidências sobre a violência doméstica e sua subnotificação durante o período de distanciamento social em decorrência da pandemia do novo coronavírus. Além disso, trabalhos futuros visando a identificar os desafios e as estratégias adotados por diferentes serviços da rede de proteção poderão fornecer informações acerca de como aconteceu o enfrentamento das situações de violência doméstica durante a referida pandemia. 


\section{Colaboradores}

M. L. Levandowski, D. N. Stahnke e R. SalvadorSilva participaram na concepção do projeto, coleta de dados, análise e interpretação dos dados, e redação e revisão crítica do estudo. T. N. Munhoz e J. V. Hohendorff participaram na interpretação dos dados e na redação e revisão crítica do estudo.

\section{Informações adicionais}

ORCID: Mateus Luz Levandowski (0000-00026188-620X); Douglas Nunes Stahnke (0000-00026871-4355); Tiago N. Munhoz (0000-0003-12819542); Jean Von Hohendorff (0000-0002-74145312); Roberta Salvador-Silva (0000-0002-7729. 7928).

\section{Agradecimentos}

Ao Departamento de Gestão de Tecnologia da Informação do Estado do Rio Grande do Sul por meio do Portal Bi Saúde, pela disponibilização pública dos dados sistematizados usados neste estudo. À CAPES conforme Portaria no 206, de 4 de setembro de 2018: "O presente trabalho foi realizado com apoio da Coordenação de Aperfeiçoamento de Pessoal de Nível Superior - Brasil (CAPES) - Código de Financiamento 001" - Bolsista Douglas Nunes Stahnke. À Caroline Krause Krug, pela dedicação e colaboração.

\section{Referências}

1. World Health Organization. Global status report on violence prevention 2014. Geneva: World Health Organization; 2014.

2. Hillis S, Mercy J, Amobi A, Kress H. Global prevalence of past-year violence against children: a systematic review and minimum estimates. Pediatrics 2016; 137:e20154079.

3. Viola TW, Salum GA, Kluwe-Schiavon B, Sanvicente-Vieira B, Levandowski ML, GrassiOliveira R. The influence of geographical and economic factors in estimates of childhood abuse and neglect using the Childhood Trauma Questionnaire: a worldwide meta-regression analysis. Child Abuse Negl 2016; 51:1-11.

4. Humphreys KL, Myint MT, Zeanah CH. Increased risk for family violence during the COVID-19 pandemic. Pediatrics 2020; 146:e20200982.

5. World Health Organization. Coronavirus disease 2019 (COVID-19): situation report, 57. Geneva: World Health Organziation; 2020.

6. União Nacional dos Dirigentes Municipais de Educação. Coronavírus: acompanhe o levantamento dos estados que suspenderam as aulas. https://undime.org.br/ noticia/17-03-2020-18-44-coronavirusacompanhe-o-levantamento-dos-estadosque-suspenderam-as-aulas- (acessado em 20/ Mar/2020).

7. Hellewell J, Abbott S, Gimma A, Bosse NI, Jarvis CI, Russell TW, et al. Feasibility of controlling COVID-19 outbreaks by isolation of cases and contacts. Lancet Glob Health 2020; 8:e488-96.

8. Fundo das Nações Unidas para a Infância. Covid-19: crianças em risco aumentado de abuso, negligência, exploração e violência em meio à intensificação das medidas de contenção. https://www.unicef.org/brazil/comunicadosde-imprensa/covid-19-criancas-em-risco-au mentado-de-abuso-negligencia-exploracao (acessado em 20/Mar/2020).

9. Secretaria de Vigilância em Saúde, Ministério da Saúde. Análise epidemiológica da violência sexual contra crianças e adolescentes no Brasil, 2011 a 2017. Boletim Epidemiológico 2018; 49(27).

10. Viodres Inoue SR, Ristum M. Violência sexual: caracterização e análise de casos revelados na escola. Estud Psicol (Campinas) 2008; 25:1121.

11. Organização das Nações Unidas. Chefe da ONU alerta para aumento da violência doméstica em meio à pandemia do coronavírus. https://nacoesunidas.org/chefe-da-onu-aler ta-para-aumento-da-violencia-domestica-emmeio-a-pandemia-do-coronavirus/ (acessado em 19/Abr/2020).

12. Campbell AM. An increasing risk of family violence during the Covid-19 pandemic: strengthening community collaborations to save lives. Forensic Science International: Reports 2020; 2:100089. 
13. Fórum Brasileiro de Segurança Pública. Violência doméstica durante a pandemia de COVID-19: 16 de abril de 2020. http://forum seguranca.org.br/wp-content/uploads/ 2018/05/violencia-domestica-covid-19-v3. pdf (acessado em 19/Abr/2020).

14. Departamento de Ações Programáticas Estratégicas, Secretaria de Atenção em Saúde, Ministério da Saúde. Diretrizes nacionais para a atenção integral à saúde de adolescentes e jovens na promoção, proteção e recuperação da saúde. Brasília: Ministério da Saúde; 2010. (Série A. Normas e Manuais Técnicos).

15. Rio Grande do Sul. Decreto no 55.129, de 19 de março de 2020. Institui Gabinete de Crise para o Enfrentamento da Epidemia COVID-19. Diário Oficial do Estado 2020; 20 mar.

16. Rio Grande do Sul. Decreto no 55.128 , de 19 de março de 2020. Declara estado de calamidade pública em todo o território do Estado do Rio Grande do Sul para fins de prevenção e de enfrentamento à epidemia causada pelo COVID-19 (novo Coronavírus), e dá outras providências. Diário Oficial do Estado 2020; 20 mar.

17. Rio Grande do Sul. Decreto no 55.115, de 12 de março de 2020. Dispõe sobre medidas temporárias de prevenção ao contágio pelo COVID-19 (novo Coronavírus). Diário Oficial do Estado 2020; 19 mar.

18. Rio Grande do Sul. Decreto no 55.154, de 1으 de abril de 2020. Reitera a declaração de estado de calamidade pública em todo o território do Estado do Rio Grande do Sul para fins de prevenção e de enfrentamento à epidemia causada pelo COVID-19 (novo coronavírus), e dá outras providências. Diário Oficial do Estado 2020; 1o abr.

19. Rio Grande do Sul. Decreto no 55.177 , de 8 de abril de 2020; Altera o Decreto no 55.154, de 1o de abril de 2020, que reitera a declaração de estado de calamidade pública em todo o território do Estado do Rio Grande do Sul para fins de prevenção e de enfrentamento à epidemia causada pelo COVID-19 (novo coronavírus), e dá outras providências. Diário Oficial do Estado 2020; 9 abr.

20. Rio Grande do Sul. Decreto no 55.184 , de 15 de abril de 2020. Altera o Decreto no 55.154, de 1 o. De abril de 2020, que reitera a declaração de estado de calamidade pública em todo o território do Estado do Rio Grande do Sul para fins de prevenção e de enfrentamento à epidemia causada pelo COVID-19 (novo coronavírus), e dá outras providências. Diário Oficial do Estado 2020; 16 abr.

21. Rio Grande do Sul. Decreto no 55.220 , de 30 de abril de 2020. Altera o Decreto no 55.154, de 1o de abril de 2020, que reitera a declaração de estado de calamidade pública em todo o território do Estado do Rio Grande do Sul para fins de prevenção e de enfrentamento à epidemia causada pelo COVID-19 (novo coronavírus), e dá outras providências. Diário Oficial do Estado 2020; 30 abr.
22. Antunes JLF, Cardoso MRA. Uso da análise de séries temporais em estudos epidemiológicos. Epidemiol Serv Saúde 2015; 24:565-76.

23. Departamento de Ações Programáticas Estratégicas, Secretaria de Atenção à Saúde, Ministério da Saúde. Linha de cuidado para a atenção integral à saúde de crianças, adolescentes e suas famílias em situação de violências: orientação para gestores e profissionais de saúde Brasília: Ministério da Saúde; 2010. (Série F. Comunicação e Educação em Saúde).

24. United Nations Womens. COVID-19 and ending violence against women and girls. https:// www.unwomen.org/en/digital-library/publica tions/2020/04/issue-brief-covid-19-and-end ing-violence-against-women-and-girls\#view (acessado em 19/Abr/2020).

25. Keenan HT, Marshall SW, Nocera MA, Runyan DK. Increased incidence of inflicted traumatic brain injury in children after a natural disaster. Am J Prev Med 2004; 26:189-93.

26. Curtis T, Miller BC, Berry EH. Changes in reports and incidence of child abuse following natural disasters. Child Abuse Negl 2000; 24:1151-62.

27. Green E, Chase RM, Zayzay J, Finnegan A, Puffer ES. The impact of the 2014 Ebola virus disease outbreak in Liberia on parent preferences for harsh discipline practices: a quasiexperimental, pre-post design. Glob Ment Health (Camb) 2018; 5:e1.

28. Marques ES, Moraes CL, Hasselmann MH, Deslandes SF, Reichenheim ME. Violence against women, children, and adolescents during the COVID-19 pandemic: overview, contributing factors, and mitigating measures. Cad Saúde Pública 2020; 36:e00074420.

29. Bourabain D, Verhaeghe PP. The conceptualization of everyday racism in research on the mental and physical health of ethnic and racial groups: a systematic review. J Racial Ethn Health Disparities 2020; [Online ahead of print].

30. Mascarenhas MDM, Sinimbu RB, Malta DC, Silva MMA, Santos AF, Vieira MLFP, et al. Violência cometida por pessoa conhecida - Brasil, 2013. Ciênc Saúde Colet 2017; 22:3763-72.

31. Witt A, Ordonez A, Martin A, Vitiello B, Fegert JM. Child and adolescent mental health service provision and research during the Covid-19 pandemic: challenges, opportunities, and a call for submissions. Child Adolesc Psychiatry Ment Health 2020; 14:19.

32. Children's Bureau, U.S. Department of Health \& Human Services. Child maltreatment 2018. Washington DC: U.S. Department of Health and Human Services; 2020.

33. Conceição MIG, Costa LF, Penso MA, Williams LCA. Abuso sexual infantil masculino: Sintomas, notificação e denúncia no restabelecimento da proteção. Psicol Clín 2020; 32:10121. 
34. Santos BR, Ippolito R. Guia escolar: métodos para identificação de sinais de abuso e exploração sexual de crianças e adolescentes. Brasília: Ministério da Educação; 2004.

35. Bavel JJV, Baicker K, Boggio PS, Capraro V, Cichocka A, Cikara M, et al. Using social and behavioural science to support COVID-19 pandemic response. Nat Hum Behav 2020; 4:460-71.

36. Keenan HT, Marshall SW, Nocera MA, Runyan DK. Increased incidence of inflicted traumatic brain injury in children after a natural disaster. Am J Prev Med 2004; 26:189-93.

37. Mohler G, Bertozzi AL, Carter J, Short MB, Sledge D, Tita GE, et al. Impact of social distancing during COVID-19 pandemic on crime in Los Angeles and Indianapolis. J Crim Justice 2020; 68:101692.

38. Fegert JM, Vitiello B, Plener PL, Clemens V. Challenges and burden of the Coronavirus 2019 (COVID-19) pandemic for child and adolescent mental health: a narrative review to highlight clinical and research needs in the acute phase and the long return to normality. Child Adolesc Psychiatry Ment Health 2020; 14:20.

39. United Nations. UN chief calls for domestic violence 'ceasefire' amid 'horrifying global surge'. https://news.un.org/en/story/2020/04/ 1061052 (acessado em 19/Abr/2020).

40. Brasil. Lei no 8.069, de 13 de julho de 1990. Dispõe sobre o Estatuto da Criança e do Adolescente e dá outras providências. Diário Oficial da União 1990; 16 jul.

41. Brasil. Lei no 8.742, de 7 de dezembro de 1993. Dispõe sobre a organização da Assistência Social e dá outras providências. Diário Oficial da União 1993; 8 dez.

42. Arruda da Silva P, Lerch Lunardi V, Dalke Meucci R, Algeri S. Protection of children and adolescents victims of violence: the views of the professionals of a specialized service. Invest Educ Enferm 2018; 36:e02.
43. Deslandes SF, Campos DS. Guardianship Councilors' views on the effectiveness of the existing network in providing full protection to children and teenagers in situations of sexual violence. Ciênc Saúde Colet 2015; 20:2173-82.

44. Faraj SP, Siqueira AC, Arpini DM. Rede de proteção: o olhar de profissionais do sistema de garantia de direitos. Temas Psicol (Online) 2016; 24:727-41.

45. Santos VA, Costa LF, Silva AX. As medidas protetivas na perspectiva de famílias em situação de violência sexual. Psico (Porto Alegre) 2011; 42:77-86.

46. Macedo DM, Foschiera LN, Bordini T, Habigzang LF, Koller SH. Systematic review of studies on reports of violence against children and adolescents in Brazil. Ciênc Saúde Colet 2019; 24:487-96.

47. Senado Federal. Proposta de Emenda Constitucional no 55 de 2016. Altera o Ato das Disposições Constitucionais Transitórias, para instituir o Novo Regime Fiscal, e dá outras providências. https://www25.senado.leg.br/ web/atividade/materias/-/materia/127337 (acessado em 19/Abr/2020).

48. Brasil. Lei no 13.431, de 4 de abril de 2017. Estabelece o sistema de garantia de direitos da criança e do adolescente vítima ou testemunha de violência e altera a Lei no 8.069/1990, de 13 de julho de 1990 (Estatuto da Criança e do Adolescente). Diário Oficial da União 2017; 5 abr.

49. United Nations Womens. Gender-based violence and COVID-19. https://www.undp. org/content/undp/en/home/librarypage/ womens-empowerment/gender-based-vio lence-and-covid-19.html (acessado em 13/ Mai/2020). 


\section{Abstract}

This study aimed to analyze the rates of reports of violence against children and adolescents in the state of Rio Grande do Sul, Brazil, from 2015 to 2020, and the changes in trends by period due to the novel coronavirus pandemic (COVID-19). This is an ecological time series study with secondary data obtained from the Bi Saúde Portal in the panel on Interpersonal Violence and Suicide. The study collected reports on violence in individuals 0 to 19 years of age in the months of March and April each year, stratified by sex, age bracket, race/color, and type of violence. Prais-Winsten regression was used to analyze the time trend. Of the 7,718 reports analyzed, there was a drop of $54 \%$ in the year 2020 compared to the same period in 2019. The analysis of the trend until 2019 indicated an increase in the reporting rates (2.04, 95\%CI: 1.01; 3.07, $p=0.002$ ), but with the inclusion of the year 2020 in the time series, the direction of the trends in reporting rates was reversed to negative, losing statistical significance (-0.39, 95\%CI: - 1.16; 2.14, $p=0.632$ ). The study concludes that social distancing due to the pandemic reduced the reporting rates of violence against children and adolescents due to underreporting, thus requiring strategies to improve the identification of suspected cases of violence during the pandemic. This highlights the need for inter-sector planning and rapid and specific actions (health, social protection, justice, and public security) in order to guarantee the rights of children and adolescents.

COVID-19; Social Isolation; Child Abuse; Domestic Violence; Pandemics

\section{Resumen}

El objetivo del estudio fue analizar las tasas de notificaciones de violencia infanto-juvenil, en el estado de Río Grande do Sul, Brasil, de 2015 a 2020, $y$ las alteraciones en sus tendencias por períodos, debido a la pandemia del nuevo coronavirus (COVID-19). Es un estudio ecológico de series temporales con datos secundarios, obtenidos mediante el Portal Bi Saúde, en la sección de Violencia Interpersonal/Suicidio. Se recogieron las notificaciones de violencia en individuos de 0 a 19 años, durante los meses de marzo y abril de cada año, estratificadas por sexo, franja de edad, raza/color y tipo de violencia. Se utilizó la regresión de PraisWinsten para el análisis de tendencia temporal. De las 7.718 notificaciones analizadas, se observó una caída de un 54\% en el año 2020, comparado con el mismo período en 2019. El análisis de tendencia hasta 2019 indicó un aumento en las tasas de notificaciones (2,04, IC95\%: 1,01; 3,07, $p=0,002$ ), no obstante, con la inclusión del año de 2020 en la serie temporal, la dirección en la tendencia de las tasas de notificaciones se invirtió en negativo, perdiendo la significación estadística (-0,39, IC95\%: $-1,16 ; 2,14, p=0,632)$. Se concluye que el confinamiento social, debido a la pandemia, redujo las tasas de notificaciones de violencia contra niños $y$ adolescentes, a causa de la subnotificación, lo que exige estrategias que mejoren la identificación de los casos sospechosos de violencia durante la pandemia. Se resalta la necesidad de planificación y acciones intersectoriales como: salud, protección social, justicia y seguridad pública, rápidas y específicas, con el objetivo de garantizar los derechos de los niños y adolescentes.

COVID-19; Aislamiento Social; Maltrato a los Niños; Violencia Doméstica; Pandemias

Recebido em 03/Jun/2020

Versão final reapresentada em 16/Out/2020

Aprovado em 06/Nov/2020 